\title{
Research on Electrical-Mechanical Energy Transfer Process of Vehicle Horn
}

\author{
Jifeng Guo, Honge Ren*, Zhiqiang Ma and Yan Ma \\ Northeast forestry university, Heilongjiang, Harbin, China \\ gif1000@126,renhonge163@163.com, zhiqiang1980@163.com, \\ myan@vip.163.com
}

\begin{abstract}
Vehicle horn is a kind of electro acoustic device. The energy transfer process were analyzed through the energetic coil current, magnetic force, and displacement curves which had gotten before, and the process was happened in stage of the action, transaction and reciprocating vibration. The reasonable criterion for checking reciprocating vibration and sounding of horn was proposed, and the criterion was proved by changed the design parameters. The conclusions are of great value for further optimizing of reliability of the electro acoustic device such as electromagnetic horn.
\end{abstract}

Keywords: Vehicle horn, vibration, electrical-mechanical energy transfer, coupled mechanical-electromagnetic characteristics

\section{Introduction}

Vehicle horn (generally for mechanical electromagnetic horn) is a kind of transducer that converts electrical energy into sound energy. The electromagnetic horn which is used for warning signal has a simple structure, low cost, easy maintenance and repair, etc. and has widely been used in water and land transportation of DC power supplying, becoming an indispensable key components of all kinds of transportation safety system [1].

Previous research focus on a single analysis to the horn diaphragm of structural dynamic characteristics [2-3].The horn of static magnetic fields distribution and suction characteristic of electromagnetic pulse is analyzed by academics [4-7], and formed the computational method of the electromagnetic coupling in the mechanical action process. The virtual prototype model of electromagnetic horns including contact breaking arc physical process was built by commercial finite element analysis software ANSYS and combined use of multi-body dynamics software ADAMS, respectively, completed the simulation analysis of the vibration characteristics and experimental tests[8-10]. In this paper, on the basis of previous research, and according to the principle of electromechanical energy conversion, mechanical electromagnetic horn was studied in power input, arc loss, resistance loss, mechanical work, magnetic field energy, kinetic energy, potential energy, sound energy from touches development phase transition to the steady stage of reciprocating vibration, and the steady state in the process of reciprocating vibration arc, arc and the cooperate relationship between electromagnetic suction and mechanical reaction, the energy conversion process in the contact closure, finally a reasonable criterion about whether electromagnetic horn can access to steady-state process of reciprocating vibration is presented. 


\section{Working Principle and Energy Relationship}

Electromagnetic horn includes dynamic iron core, static iron core, the excitation coil and plate shell, etc. Contact system includes dynamic contact, moving contact spring, static contact, static reed and insulating diaphragm [8]. Diaphragm and the trumpet constitute the vocal vibration parts, as shown in Figure 1.

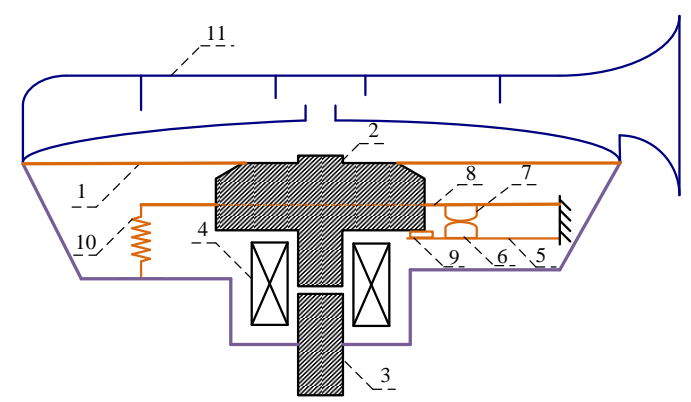

1. Diaphragm; 2. Dynamic Iron Core; 3. Static Iron Core; 4. Coil; 5. Dynamic Reed; 6. Dynamic Contact; 7. Static Contact; 8. Static Reed; 9. Insulating Diaphragm; 10. Screw; 1. Trumpet

\section{Figure 1. Simple Construction Diagram of Electromagnetic Horn}

Coil power on the DC current can generate magnetic field, the electromagnetic force driving dynamic core and diaphragm move downward, then dynamic iron core promote the normally closed contact breaking, excitation current reduce to zero. When electromagnetic suction is lower than the reaction force generated by the spring and diaphragm mechanical structure generated the move iron core will move from bottom to top by the reaction force. The contact recovering closed state and conducing excitation coil current which generate the magnetic force again and attracting dynamic iron core repeat the above process. In this process the diaphragm generates harmonic vibration under periodic external force, cause the surrounding air to fluctuate, generate sound and spread out by the horn.

Seeing the electromagnetic horn as a two port device: mechanical port and electrical port. Shifting out the resistance loss and mechanical loss, respectively with the coil resistance R and arc resistance Rc (zero when the arc extinguish, contact resistance when contact is closed), moving drag coefficient, and regardless of the dielectric loss of ferromagnetic medium, the device center will become a lossless core, air gap and without copper loss, mechanical loss and dynamic coupling coil of lossless magnetic energy storage system, as shown in Figure 2.

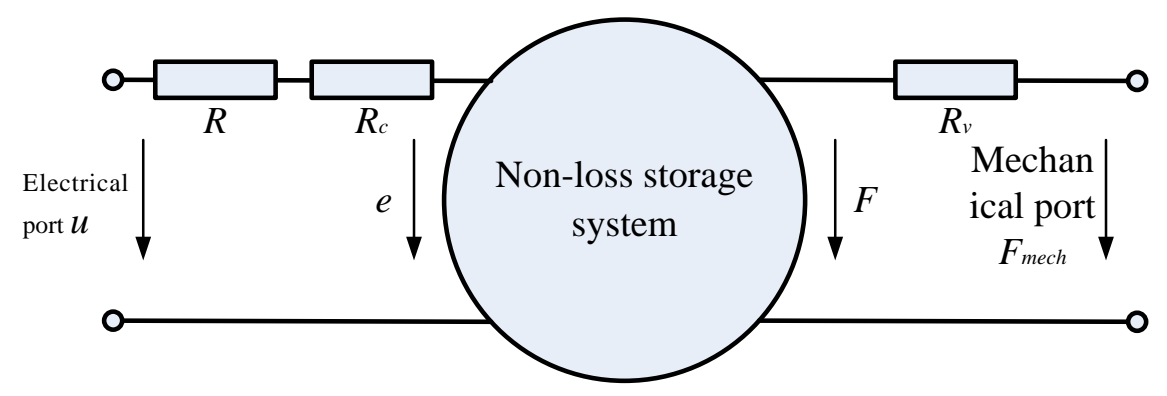

Figure 2. Schematic of Mechanical Energy and Electromagnetic Energy Conversion Process 
As shown in Figure2, for single incentive non-loss storage system, in time $\mathrm{d} t$, the power input is

$$
\mathrm{d} W_{e}=E i \mathrm{~d} t=i \mathrm{~d} \psi+i^{2}\left(R+R_{c}\right) \mathrm{d} t
$$

The change of coupling field magnetic energy is

$$
\mathrm{d} W_{m}=\frac{\partial W_{m}}{\partial \psi} \mathrm{d} \psi+\frac{\partial W_{m}}{\partial y_{\text {mech }}} \mathrm{d} y_{\text {mech }}=i \mathrm{~d} \psi-F_{e} \mathrm{~d} y_{\text {mech }}
$$

Mechanical energy output is $\mathrm{d} W_{\text {mech }}=F_{e} \mathrm{~d} y_{\text {mech }}$, for coupling field, ordering input energy is positive, the output is negative. According to the above relations, the magnetic energy $\mathrm{d} W_{m}$ increment includes two parts: the magnetic energy increment caused by the flux change $\frac{\partial W_{m}}{\partial \psi_{1}} \mathrm{~d} \psi_{1}$, equaling the difference between the power absorbed from net power $\mathrm{d} W_{e}$ and the energy consumption of resistance; the magnetic energy increment caused by changes in displacement $\frac{\partial W_{m}}{\partial \theta} \mathrm{d} \theta$, equaling the output of the differential mechanical negative energy $\mathrm{d} W_{\text {mech }}$. In the process of energy conversion, as a coupling magnetic field can be either input or output energy from electrical system, also can be either output or input energy for mechanical system, its state mainly depends on the constraints of the magnetic chains $\psi$ and displacement of moving part $y$ [11].

Moving iron core and the vibration system composed of diaphragm meet the principle of conservation of energy under the action of external mechanical force in the process of the reciprocating vibration, external do mechanical work with the system kinetic energy and elastic potential energy and the output of the sound energy (energy dissipation of air damping n) keep conservation. $F_{e} \mathrm{~d} y_{\text {mech }}$ can be rewrite into electromagnetic horns' mechanical system of kinetic energy and elastic potential energy (diaphragm, moving contact spring), and the change of acoustic emitted energy, Accordingly type (2) can be rewritten as

$$
i \mathrm{~d} \psi=\mathrm{d} W_{m}+\mathrm{d} W_{\text {kinetic energy }}+\mathrm{d} W_{\text {potential energy }}+\mathrm{d} W_{\text {sound energy }}
$$

$W_{\text {kinetic energy }}$ is the sum of the kinetic energy of the moving iron core, diaphragm and the moving contact spring, namely $W_{\text {kinetic energy }}=\sum_{i} \frac{1}{2} m_{i} v_{i}^{2} ; W_{\text {potential energy }}$ is the sum of elastic potential energy of diaphragm and moving contact spring, namely $W_{\text {potential energy }}=\sum_{i} \frac{1}{2} k_{i} y_{i}^{2}$.

\section{Mechanical and Electrical Energy Conversion Process}

As shown in Figure 3 according to the simulation of correct virtual prototype model of electromagnetic horn, we can analysis to get the curve of voice coil current, electromagnetic force, the moving iron core displacement and the dynamic contact displacement, in the process from the coil electrifying to stable as a result. Complete working process can be divided into the touch, transient and steady state process of reciprocating vibration [6]. 


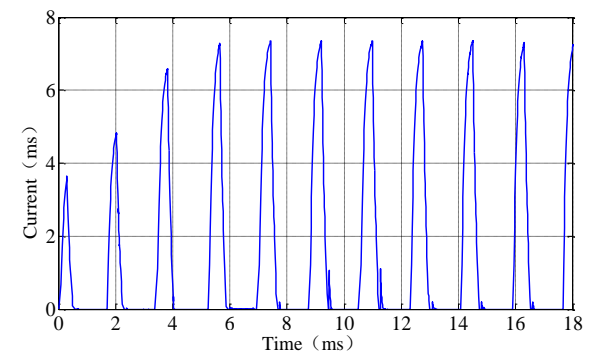

(a) Dynamic Current

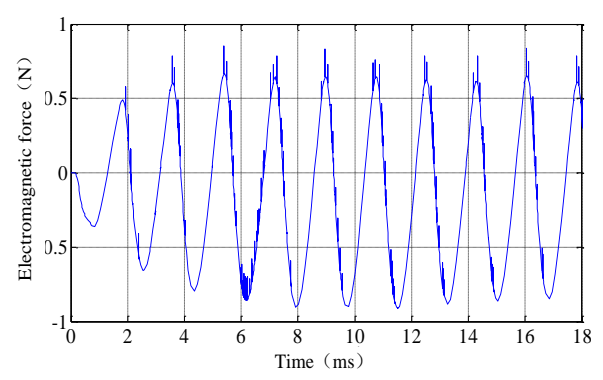

(c)Moving Iron Core Displacement

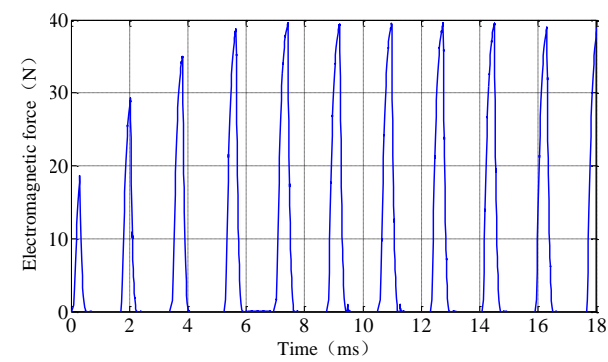

(b) Electromagnetic Force

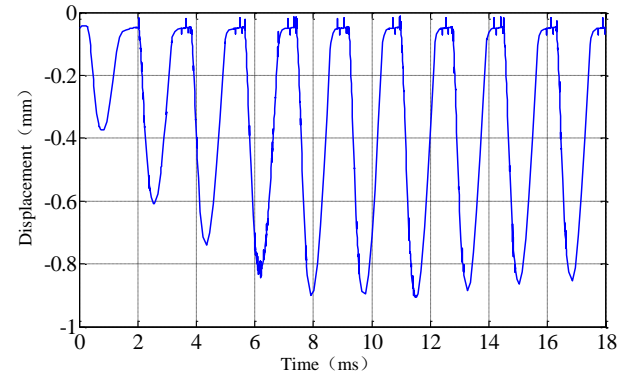

(d) Dynamic Contact Displacement

Figure 3. Simulation Results by Using Virtual Prototype

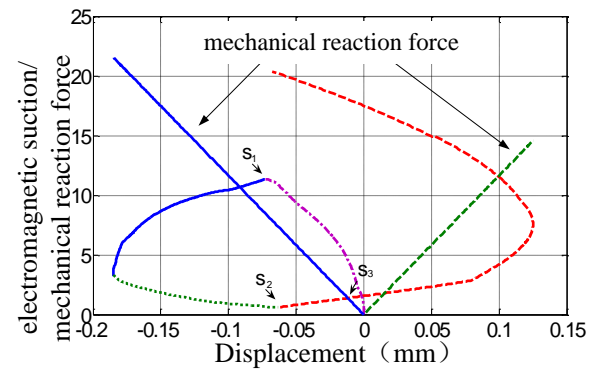

(a) First Cycle

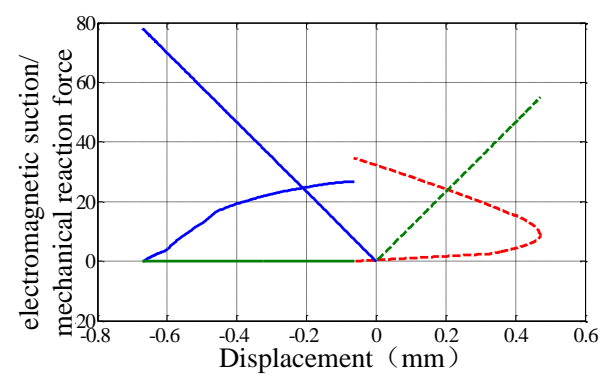

(c)Third Cycle

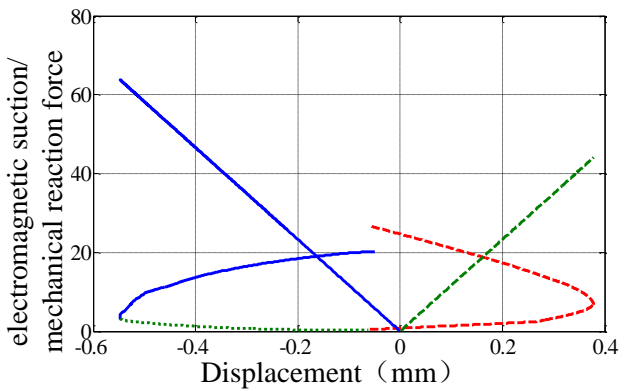

(b)Second Cycle

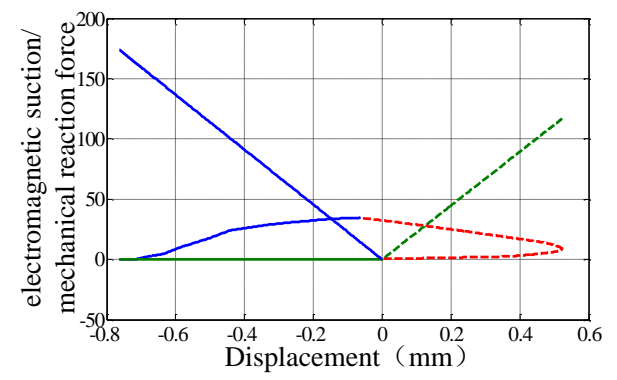

(d) Forth Cycle

Figure 4. Relationship Between Attraction Force and Mechanical Force During Whole Operation

Horns can achieve stable reciprocating cycle vibration, which is inseparable energy accumulation in the process of early touches and transition. As shown in Figure 4 is the time-varying cooperate relationship between dynamic electromagnetic suction and mechanical reaction force, including the process of touches, transient and steady reciprocating vibration state of mechanical electromagnetic horn. 
The stage of touches coil began to electricity, and the electromagnetic force is greater than the mechanical reaction force, thus leading moving iron core drives the diaphragm downward movement. In the process of transition, the displacement of moving iron core and diaphragm can be represented as $y_{c}$, the mechanical work of electromagnetic suction in this period of time can be represented as, $\int_{0}^{y_{c}} F_{1} \mathrm{~d} y$, the diaphragm elastic potential energy can be represented as $\int_{0}^{y_{c}} k y \mathrm{~d} y$. Now, the kinetic energy of the moving iron core is

$$
W_{1 d}=\int_{0}^{y_{c}}\left(F_{1}-k y\right) \mathrm{d} y
$$

If initial pressure of spring system is $F_{0}$, the elastic potential energy of spring can be expressed as

$$
W_{p}=\frac{1}{2} k_{c} y_{20}^{2}
$$

$k_{c}--$ the spring equivalent stiffness;

$y_{20}$-- the deformation of spring in contact location.

When $W_{1 d}>W_{p}$, the moving iron core with the rest of the kinetic energy can make contact into the arc state. At this time, electromagnetic suction of $12 \mathrm{~N}$, by the reference paper [6], stable energy conversion mechanism of a reciprocating motion process, during the arc ,the electromagnetic force do positive work for mechanical system and makes displacement amplitude of moving iron core gradually increasing, and makes the moving iron core upward movement cross the initial position in the return recoil and makes contact closure time prolong, providing electromagnetic force continue doing positive work to mechanical systems. After the point $s_{1}$, electromagnetic suction will less than mechanical reaction force, reducing moving iron core movement speed. As shown in Figure 4 (a), upward return movement exists between point $s_{2}$ and point $s_{3}$, where electromagnetic suction less than the mechanical reaction stage, but with previously stored kinetic energy smoothly completed through $s_{3}$ where electromagnetic suction is greater than the mechanical reaction again. This cycle the downward movement displacement amplitude of moving iron core is $0.17 \mathrm{~mm}$, the corresponding mechanical reaction force is to $21 \mathrm{~N}$, the upward movement displacement amplitude of moving iron core is $0.12 \mathrm{~mm}$, the corresponding mechanical reaction force is to $15 \mathrm{~N}$.

When moving iron core second run into spring making contacts breaking, it has a bigger coil current than the first time, so the electromagnetic suction increased to $20 \mathrm{~N}$. As shown in Figure 4 (b), this cycle the downward movement displacement amplitude of moving iron core is $0.57 \mathrm{~mm}$, the corresponding mechanical reaction force is to $63 \mathrm{~N}$, the upward movement displacement amplitude of moving iron core is $0.38 \mathrm{~mm}$, and the corresponding mechanical reaction force is to $42 \mathrm{~N}$.

When moving iron core third run into spring making contacts breaking, it has a bigger coil current than the second time, so the electromagnetic suction increased to $28 \mathrm{~N}$. As shown in Figure 4 (c), this cycle the downward movement displacement amplitude of moving iron core is $0.7 \mathrm{~mm}$, the corresponding mechanical reaction force is to $78 \mathrm{~N}$, the upward movement displacement amplitude of moving iron core is $0.48 \mathrm{~mm}$, and the corresponding mechanical reaction force is to $54 \mathrm{~N}$.

When moving iron core forth run into spring making contacts breaking, it has a bigger coil current than the third time, so the electromagnetic suction increased to $36 \mathrm{~N}$. As shown in Figure 4 (d), this cycle the downward movement displacement amplitude of 
moving iron core is $0.8 \mathrm{~mm}$, the corresponding mechanical reaction force is to $170 \mathrm{~N}$, the upward movement displacement amplitude of moving iron core is $0.5 \mathrm{~mm}$, the corresponding mechanical reaction force is to $115 \mathrm{~N}$. The electromagnetic horn has entered a steady process of reciprocating vibration.

Figure 5 is corresponding closing time in the process. Touched stage which is completed by static acceleration process, thus the closing time is the longest $: 2.7 \mathrm{~ms}$. During the first cycle in the transitional process, contact has the first acceleration and second deceleration characteristics, the closing time dropped to $0.7 \mathrm{~ms}$. In the second cycle, the contact closure time increase $0.2 \mathrm{~ms}$ than first cycle, in the third cycle, the contact closure time increased $0.1 \mathrm{~ms}$, eventually in the steady state stage the closure time is stability to $1 \mathrm{~ms}$. In the transition process, the recoil distance which from upward return movement of moving iron core is constant improvement, providing the contact closure time increased; therefore extended the rebuilding process of coil current, making electromagnetic suction in the process of touches and transition increase.

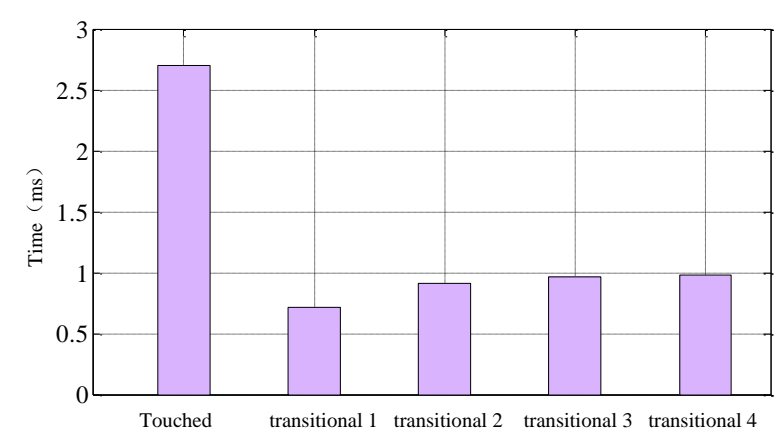

\section{Figure 5. The Rules of Keeping Contact Time}

From Figure 3 we can see in the first two motion cycle the clearance between open contacts did not reach the maximum, electric arc not fully burning then enter the closed state. Through two cycles, the mechanical work injects energy into mechanical system and makes it get store energy accumulation. The moving iron core movement amplitude increase, causing the electromagnetic force and mechanical work which electromagnetic force do gradually increases, the moving iron core displacement amplitude increase which will make the whole system stored potential energy and the convertible kinetic energy gradually increases, until the single cycle the mechanical work of which electromagnetic force do equal damping energy dissipation, so as to achieve stable process of reciprocating vibration, the displacement amplitude of moving iron core is constant, potential energy and convertible kinetic energy of storage system keep conservation, the mechanical work input and output of the sound energy is constant. Figure 6 is the transformation function relation of electromagnetic horn in transition. 


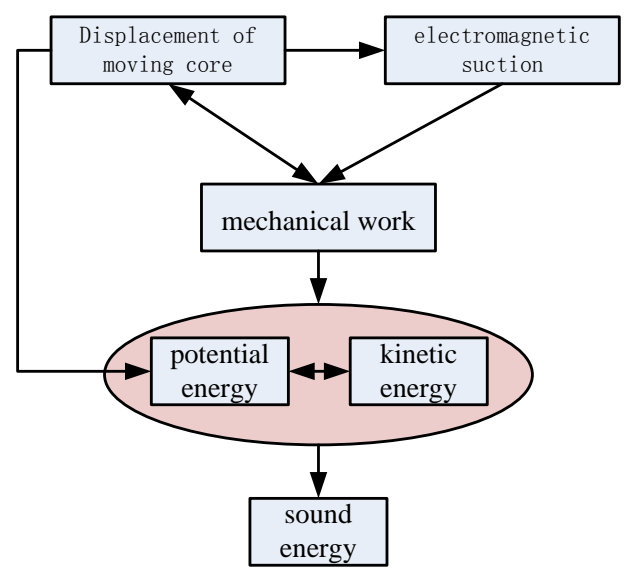

\section{Figure 6. The Conversion Relationship Between Work and Energy During Transition Phase}

For traditional electrical components with relay characteristics, it require the action of voltage (or current) electromagnetic suction higher than that of mechanical reaction force everywhere, the release voltage (current) electromagnetic suction should lower than mechanical reaction force. For electromagnetic horns, the reciprocating vibration stable state is the basic guarantee of its steady voice. Figure 4 shows, there is many times change on numerical value and direction of dynamic electromagnetic suction and mechanical reaction in the all the work process, and sometimes the dynamic electromagnetic suction above mechanical reaction force, and other times the dynamic electromagnetic suction under mechanical reaction force. Apparently these principles do not apply to judge whether the electromagnetic horn can realize reciprocating vibration stability.

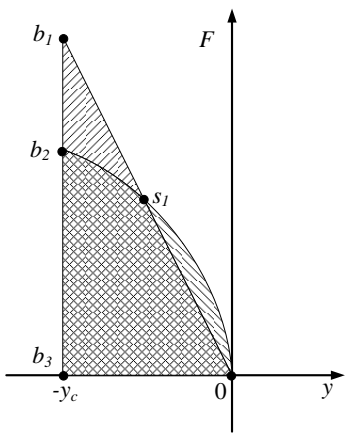

Figure 7. Schematic Diagram of Mechanical Work during Actuation Process

Through applying the principle of energy conversion, we known that electromagnetic horn have the adaptive adjustment features during touches and transition process, namely, which the system needn't impressed current incentives adjustment and transformation, it can also realize that electromagnetic horn conversion from touches process to transition to stable and develop reciprocating vibration. Touch process is a preparation of transition process and is also deciding whether mechanical electromagnetic horn realizes final reciprocating stable vibration. The comparison between the mechanical work which dynamic electromagnetic suction do in the process and the mechanical potential energy consumed by mechanical reaction force decide whether the moving iron core can enter the transition process. Figure 7 shows, dynamic electromagnetic suction and mechanical reaction force equilibrium point is $s_{1}$. The mechanical work which electromagnetic suction does in the touches process is the area $S_{o b_{2} b_{3}}$, and the elastic 
potential energy which the mechanical reaction force cost is the area $S_{\overline{o b_{1} b_{3}}}$. So if area $S_{o b_{2} b_{3}}$ equal area $S_{\overline{o b_{1} b_{3}}}$ or area $S_{o b_{2} b_{3}}$ is smaller than the area $S_{\overline{o b_{1} b_{3}}}$, then the kinetic energy of the moving iron core is zero in $y_{c}$ and the velocity is also zero, thus it can't separate the contact arc and the moving iron core can not enter the transition process. If area $S_{o b_{2} b_{3}}$ is greater than the area $S_{\overline{o b_{1} b_{3}}}$, the kinetic energy of the moving core is more than zero in $y_{c}$,guaranteeing the moving iron core is a state of accelerated motion into arc stage, and the mechanical work at this time can make its complete the energy accumulation of mechanical system to enter transition process and stability of reciprocating vibration. Usually taking into account which the reliability design factors need to leave enough margins, so you can determine use the balance point $s_{1}$ which appears in the stage of arc (including location $y_{c}$ ) as the necessary conditions which mechanical electromagnetic horn can develop to ensure entering reciprocating vibration.
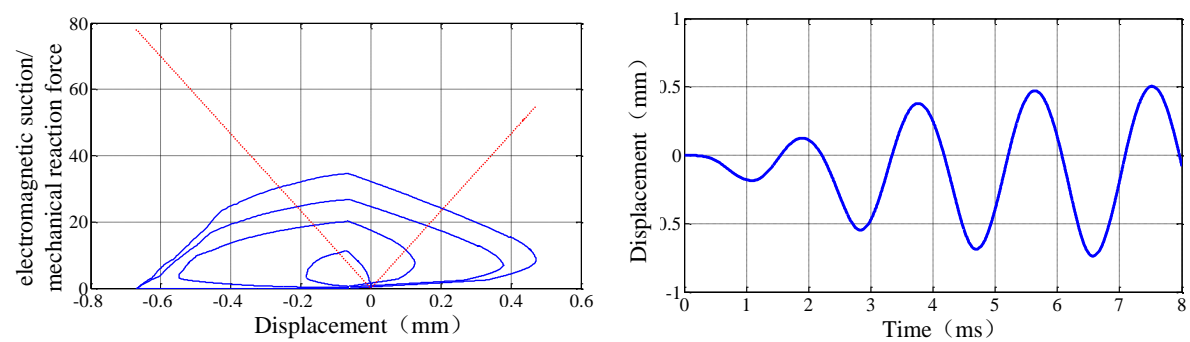

Figure 8. Results of $k=116 \mathrm{~N} / \mathrm{mm}$
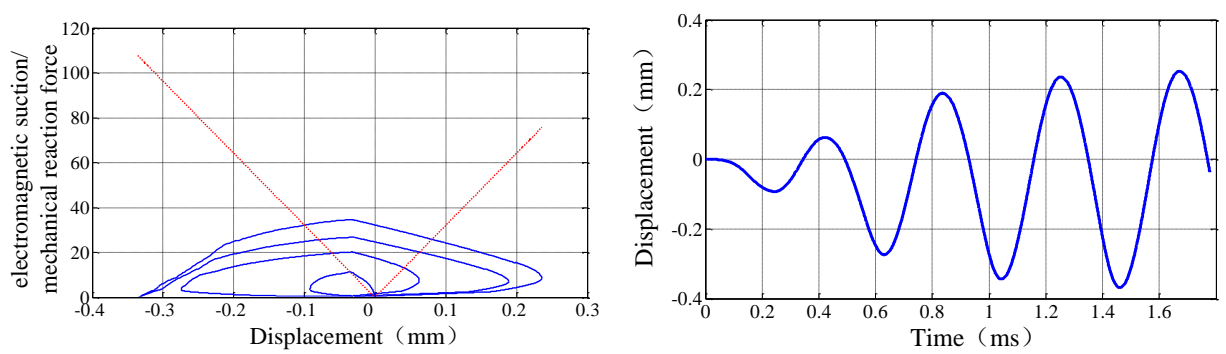

Figure 9. Results of $k=322 \mathrm{~N} / \mathrm{mm}$

Reference paper [10] has pointed out that mechanical force can be adjust by changing the stiffness of diaphragm. Figure $8 \sim 10$, respectively corresponding to the simulation analysis results of the moving iron core of electromagnetic horn in touches and transition process under the different diaphragm stiffness condition. When diaphragm stiffness $k=116 \mathrm{~N} / \mathrm{mm}$, equilibrium point $s_{1}$ occurs in the arc stage, after three system transition cycles, the vibration amplitude and electromagnetic suction achieves stable cycle condition. When diaphragm stiffness $k=322 \mathrm{~N} / \mathrm{mm}$, dynamic electromagnetic suction equal mechanical reaction force in location $y_{c}$, the system can still reach stabilization the reciprocating vibration, the maximum value of electromagnetic suction increased compared with the previous one, but the amplitude is smaller. When diaphragm stiffness $k=500 \mathrm{~N} / \mathrm{mm}$, the scope of the moving iron core is within $[0 \sim 0.009 \mathrm{~mm}$, the dynamic electromagnetic suction is greater than the mechanical reaction force in this area, that it will leads accelerated motion; Within $[0.009 \mathrm{~mm} \sim 0.013 \mathrm{~mm}]$, the velocity will 
slow down to zero, then the moving iron core return movement due to the reaction effects of the elastic potential energy, and ultimately it will be stable oscillation in the equilibrium position $0.009 \mathrm{~mm}$, the moving iron core don't contact with spring, in the process, thus determine electromagnetic horns don't enter the process of transition, the phenomenon referred to as the "vibration" state.
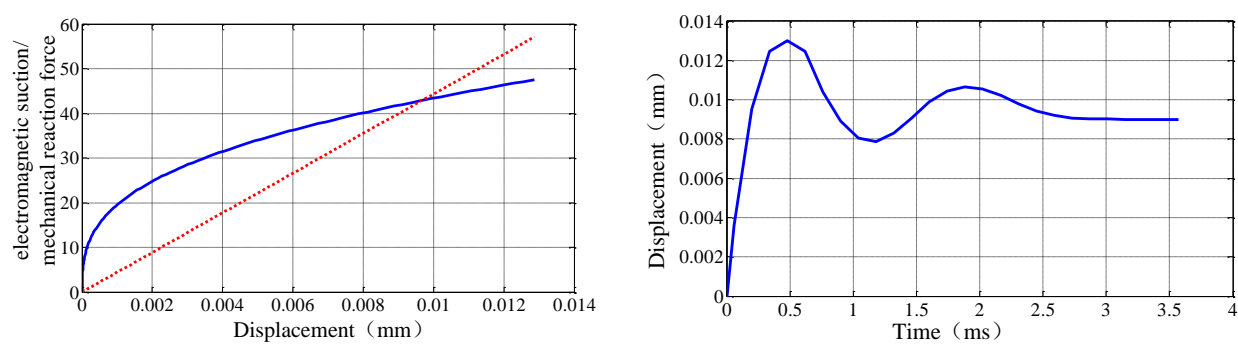

Figure 10. Results of $k=500 \mathrm{~N} / \mathrm{mm}$

\section{Conclusions}

In this paper, according to the principle of electromechanical energy conversion, studied energy conversion relations of vehicle horn from the touches to the steady reciprocating vibration state, laying a foundation for optimization design of the electromagnetic horn.

(1)Using the dynamic change rule of performance parameters through the simulation analysis as the foundation, analysis the dynamic matching relations between the electromagnetic suction and mechanical reaction force, fully analyzed physical process of the electromagnetic horn from touches to steady reciprocating vibration .

(2)Put forward a reasonable criterion about whether the electromagnetic horn can enter the steady reciprocating vibration or not, namely the equilibrium point ${ }^{S_{1}}$ (including location ${ }_{c}$ ) of the dynamic electromagnetic suction and mechanical reaction force locate in the phase of arc is the necessary conditions which the mechanical electromagnetic horn can develop to reciprocating vibration. Meet the conditions of electromagnetic horn has the ability of self-adjusting during the transition process, namely through the mechanical work complete system energy accumulation stored until reach steady-state reciprocating vibration, mechanical work will be converted to sound energy output (damping energy dissipation) at this time.

\section{Acknowledgements}

This work was supported by the Fundamental Research Funds for the Central Universities (2572014CB27) and the science and technology project of Heilongjiang Province Education Department No. 12533029

\section{Reference}

[1] Guo J. F., Ren W. B., Zhai G. F., "J. Technological Development of Automobile Electric Horn," Electromechanical Components, vol. 1 no. 30, (2010), pp. 39-46.

[2] Ye Y. W., Wang Z. L., Pan J. J., "J. Study on Acoustic Characteristic of Helicoid Electric Horn Influenced by the Horn Film Vibration," Vibration and Shork, vol. 2 no. 20, (2001), pp. 56-58.

[3] Ji Q., Jiang W. K., Qi F. H., "J. Analysis Of Affect Factors For Life Cycle Of Horn Diaphragm," Vibration and Shork, vol. 5 no. 26, (2007), pp. 112-114.

[4] R. Ravaud, G. Lemarquand and T. Roussel, "J. Time-varying nonlinear modeling of electrodynamic loudspeakers," Applied Acoustics, vol. 3 no. 70, (2009), pp. 450-458.

[5] Guy L., "Ironless Loudspeakers," J.IEEE Transactions on Magnetics, vol. 8 no. 43, (2007), pp. 33713374. 
[6] R. Rashedin and T. Meydan, "J. Solenoid actuator for loudspeaker application," Sensors and Actuators A, vol. 5 no. 129, (2006), pp. 220-223.

[7] R. Ravaud, G. Lemarquand and T. Roussel, "J. Time-varying nonlinear modeling of electrodynamics loudspeakers," Applied Acoustics, vol. 7 no. 70, (2009), pp. 450-458.

[8] Guo J. F., Ren W. B., Zhai G. F., "J. Cycle Vibration Characteristics and Energy Transfer Mechanism of Electromagnetic Horn," Transactions of China Electrotechnical, vol. 3 no. 26, (2011), pp. 74-79.

[9] Guo J. F., Ren W. B., Zhai G. F., "J. A Research on the Simulation Method for Coupled Mechanical Electric Magnetic and Acoustic Fields for Automotive Horn," Automobile Engineering, vol. 5 no. 33, (2011), pp. 452 456.

[10] Guo J. F., Ren W. B., Zhai G. F., "J. Piecewise linear vibration of an electromagnetic horn and its acoustic characteristics," Vibration and Shork, vol. 10 no. 30, (2011), pp. 224-227.

[11] Tang Y. Q., "Electric Machinery (The Fourth Edition)," China Machine Press, vol. 3, (2011) (2011), pp. 309-327.

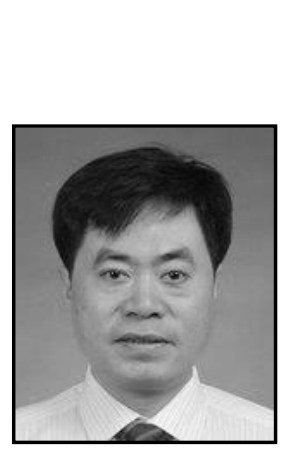

\begin{abstract}
Authors
Jifeng Guo, he was born in 1974. He received the Ph.D. degree from Harbin Institute of Technology, China, in 2011. He is currently an associated professor of information and computer engineering college at School of Northeast Forestry University. His main research interests include the reliability theory simulation techniques of Vehicle electrical appliance.
\end{abstract}

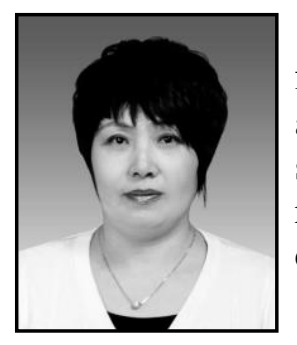

Honge Ren, she was born in 1962. She received the Ph.D. degree from Northeast forestry university, China, in 2009. She is currently a professor of information and computer engineering college at school of Northeast forestry university, supervisor of Dr. Her main research interests include the pattern recognition and intelligent control.

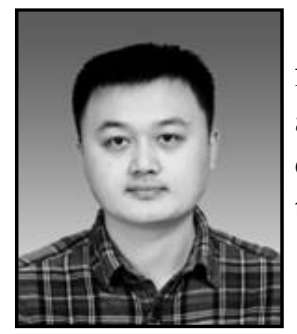

Zhiqiang Ma, he was born in 1980. He received the master degree from Northeast forestry university, China, in 2009. He is currently an lecturer of information and computer engineering college at school of Northeast forestry university. His main research interests include the pattern recognition and intelligent control.

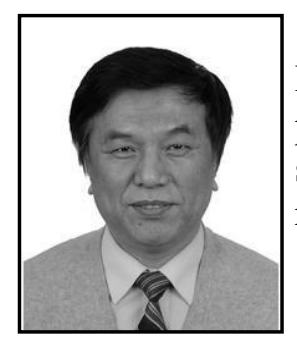

Yan Ma, he was born in 1955. He received the master degree from Harbin Institute of Technology, China, in1985. He is currently an professor of college of mechanical and electrical engineering at school of Northeast forestry university, supervisor of Dr. His main research interests are mechanism design theory. 